Ebisu

Études japonaises

Études japonaises

48 automne-hiver 2012

Naissance d'une revue féministe au Japon : Seitō

(1911-1916)

\title{
La chasteté, d'un devoir vers un droit, au prisme du débat (1914-1916) autour de Seitō
}

Chastity, From a Duty to a Right, Through the Debate (1914-1916) About Seitō

貞操義務から貞操権へ—『青鞜』における論争（1914-1916年）をめぐ

っ

Isabelle Konuma

\section{CpenEdition}

1 Journals

Édition électronique

URL : http://journals.openedition.org/ebisu/658

DOI : 10.4000/ebisu.658

ISSN : 2189-1893

Éditeur :

Institut français de recherche sur le Japon (UMIFRE 19 MAEE-CNRS), Maison franco-japonaise

Édition imprimée

Date de publication : 1 septembre 2012

Pagination : 147-166

ISSN : $1340-3656$

\section{Référence électronique}

Isabelle Konuma, "La chasteté, d'un devoir vers un droit, au prisme du débat (1914-1916) autour de Seitō », Ebisu [En ligne], 48 | automne-hiver 2012, mis en ligne le 21 mai 2014, consulté le 01 mai 2019. URL : http://journals.openedition.org/ebisu/658 ; DOI : 10.4000/ebisu.658 


\title{
La chasteté, d'un devoir vers un droit Au prisme du débat (1914-1916) autour de Seitō
}

\author{
Isabelle KONUMA*
}

La publication, en septembre 1914, d'un article d'Ikuta Hanayo 生田花世 (1888-1970) ${ }^{1}$ dans Hankyō 反響 (Résonance) marqua le début d'un débat qui dura près de deux ans. Dans cet article, Ikuta Hanayo déclara qu'elle avait renoncé à sa virginité pour subvenir aux besoins de sa famille en cédant aux avances de son employeur, ce qui suscita une réaction virulente, notamment chez Yasuda (Harada) Satsuki 安田(原田)㿞月 (1887-1933) ${ }^{2}$ qui publia une réponse dans Seitō 青鞜 (Les Bas-bleus) en décembre de la même année ${ }^{3}$. Un débat s'ensuivit entre les deux femmes, auquel participèrent très vite les deux principales animatrices de Seitō, Itō Noe 伊 藤野枝 (1895-1923), en février 1915, puis Hiratsuka Raichō 平塚らいてう

* CEJ-INALCO.

1. Née à Tokushima, elle fut enseignante jusqu'au décès de son père. Elle décida alors de monter à Tokyo où elle ne cessa d'écrire tout en s'essayant à divers emplois. Elle vécut en couple avec le poète Ikuta Shungetsu 生田春月 de 1914 à 1930, jusqu'au suicide de celui-ci. Elle signe ses premiers textes Nishizaki 西崎 dans Seitó (voir l'article d'Ōta Tomomi dans ce même dossier).

2. Née dans une famille d'éducateurs à Niigata, elle se maria avec un musicien, Harada Jun 原田潤, en 1915, après avoir vu ses fiançailles avec un autre homme annulées pour cause de pneumonie. Elle prôna le travail et l'indépendance financière des femmes, et devint responsable de l'Association de la femme nouvelle (Shinfujin kyōkai 新婦人協会) d'Osaka. Elle mit fin à ses jours en 1933, suite à une nouvelle crise de pneumonie.

3. Les articles constituant le débat sur la chasteté sont rassemblés dans Orii (1991). 
(1886-1971) en mars. En avril, Ōsugi Sakae 大杉栄 (1885-1923) ${ }^{4}$ y fit son apparition en réponse à Itō Noe, ainsi que Yosano Akiko 与謝野晶子 (1878$1942)^{5}$, puis différentes personnalités y apportèrent leur contribution. Le débat parut dans plusieurs revues ${ }^{6}$, en débutant sur une interrogation simple : une femme doit-elle dépendre d'un homme pour se nourrir ? La signification du mariage et le sens de la prostitution furent passés au peigne fin, ce qui mena aux thèmes centraux, que sont la nature de la chasteté en tant que règle morale, et l'inégalité sexuelle en son sein.

S'étalant sur deux ans (1914-1916), ce débat traita principalement de la question de l'émancipation de la femme sur le plan sexuel, bien avant la deuxième vague du féminisme qui s'y attaqua dans les années soixante en termes de droit à la reproduction, critique de la commercialisation du sexe, etc. ${ }^{7}$. Il faut à ce titre rappeler l'influence qu' eut sur Hiratsuka Raichō l'ouvrage d'Ellen Key (L'Amour et le mariage, 1904) où furent développées des critiques de la morale chrétienne traditionnelle en faveur d'un mariage d'amour et de la liberté dans le divorce ainsi que la reproduction ${ }^{8}$. Suivi de deux autres débats sur la prostitution et sur l'avortement, animés toujours autour de Seitō, la chasteté ouvrit ainsi la voie à une reconsidération de

4. Né dans une famille de militaires, il fut fortement influencé par les mouvements pacifistes et socialistes de Kōtoku Shūsui 幸徳秋水 (1871-1911), et devint par la suite un fervent activiste anarchiste. En 1923, il fut assassiné par la gendarmerie (kenpeitai 憲 兵隊), avec Itō Noe, sa compagne de l'époque, ainsi que son neveu.

5. Poétesse et écrivaine, elle fut aussi une fervente militante pour l'amélioration des conditions féminines (voir Dodane 2000).

6. Outre les deux revues citées, plusieurs revues y consacrèrent leurs pages : Shinkōrōn 新公論 (Nouveaux débats), Fujo shinbun 婦女新聞 (Journal des femmes), Daisan teikoku 第三帝国 (Le troisième empire), Fujo shinpo 婦女新報 (Nouvelles des femmes) et Fujin kōron 婦人公論 (Débat public des femmes) à la fin, en 1916.

7. Dès les premières années de Meiji, des débats furent entrepris sur la sexualité en relation avec la question de la prostitution ou de la monogamie, notamment au sein de la Société de l'an 6 (Meiroku-sha 明六社) avec Mori Arinori 森有礼 (1847-1889), Fukuzawa Yukichi 福澤諭吉 (1834-1901) ou Nakamura Masanao 中村正直 (18321891). Quant au débat sur la chasteté, tout en présentant des traits novateurs, il s'inscrit dans le prolongement des échanges qui eurent lieu autour de l'égalité sexuelle, notamment entre les juristes au moment de la rédaction du Code civil (1898), ou au sein du Mouvement pour la liberté et les droits du peuple (Jiyū minken undō 自由民権運動).

8. Voir les articles de Christine Lévy et d'Ōta Tomomi dans ce dossier. 
l'ordre social et intime des femmes ${ }^{9}$. Or, contrairement aux deux autres débats, la chasteté semble poser un problème préalable, à savoir la définition des termes. En effet, les débats souffrent d'une absence de définition unanime et précise du mot teisō 貞操 (chasteté), tantôt remplacé par shojo 処女, tantôt remplacé par dōtei 童貞 ${ }^{10}$, mettant en évidence une absence de cadre de réflexion commun.

Afin de comprendre comment un tel débat naquit précocement, et comment celui-ci peut être situé dans l'évolution sociale et institutionnelle, dans le présent article, il sera d'abord proposé une analyse de la conception de la chasteté selon les auteurs, puis une interprétation de ces débats d'un point de vue juridique. Pour cela, l'analyse sera faite principalement à l'aide d'ouvrages et d'articles juridiques de la fin de Meiji jusqu'au début de l'ère Shōwa.

\section{1. Élaboration d'un cadre de réflexion commun autour de la chasteté}

La complexité, voire la confusion des auteurs dans l'usage du concept de chasteté nous posa rapidement un problème dans le cadre d'un travail de traduction des articles de Seitō. En effet, très vite, le mot teisō se révéla problématique faute d'équivalent en français, le mot, traduit principalement par " chasteté ", devant être remplacé, selon les passages, par "virginité " pour respecter une cohérence d'ensemble. Ce travail fut d'autant plus périlleux que, par endroit, les auteures, elles-mêmes dans une confusion conceptuelle, revinrent plus tard pour corriger leur erreur.

Le débat commence avec un article d'Ikuta Hanayo, publié en septembre 1914 dans Hankyo ${ }^{-11}$. Dans cet article, l'auteur explique qu'elle dut renoncer à sa chasteté pour subvenir aux besoins de son petit frère, et que ce choix était finalement inévitable, relevant de la " nature des choses». Elle se réjouit plutôt d'avoir pris conscience du lien indissoluble pour les femmes

9. Sur la formation d'une nouvelle identité féminine à travers l'amour, la sexualité et le mariage, voir Suzuki (2010). Voir également l'article introductif du même dossier.

10. Aujourd'hui, shojo désigne une femme vierge alors que dōtei désigne la virginité de l'homme.

11. Ikuta Hanayo, 1914, «Taberu koto to teisō to » 食べることと貞操と (Manger et rester chaste), Hankyō, septembre 1914 (Orii 1991 : 13-18). 
entre manger et rester chaste, puisque les femmes "ne peuvent détenir de patrimoine selon le système juridique en vigueur ». Yasuda Satsuki y répond avec un ton très ferme, se sentant presque insultée dans sa nature féminine $^{12}$ : pour elle, la chasteté a un sens bien plus large que la simple virginité, elle constitue l'intégrité féminine, la raison d'être des femmes. La chasteté n'est pas non plus la fidélité d'une femme mariée à son époux, cette fidélité - du moment où elle n'est pas soutenue par l'amour - n'étant qu'un camouflage d'une forme de prostitution qui garantirait à la femme sa propre subsistance.

Les premières pierres étant ainsi posées, Ikuta Hanayo revint sur la notion de chasteté, en précisant qu'elle signifiait, du moins dans son premier article, "virginité ", en employant pour la première fois les termes shojo et dōtei ${ }^{13}$. Or, la virginité "n'a pas de propriétaire »; la chasteté, elle, appartient à l'un et à l'autre dans un couple lié par l'amour. Ikuta Hanayo reste cependant peu convaincante quand elle dit assumer ce choix, avoir renoncé à sa virginité "en connaissance de cause », recourant simplement à son droit de disposer de son corps, et ce par amour pour son petit frère. Or, dans un autre passage, elle avoue que ce choix était en quelque sorte un " choix subi ", puisque l'entière responsabilité reviendrait à la société qui ne lui en laissait pas d'autre. Pour Ikuta, l'amour est fondamental entre un homme et une femme, la chasteté en résulte naturellement tant qu'amour il y a. Ainsi, le sacrifice de la chasteté ne pourrait pas être utilisé comme un moyen de subsistance si une femme aime un homme, la prostitution étant un moyen de subsistance malheureux des femmes sans conjoint. Elle dissocie par conséquent la virginité de la chasteté d'une femme engagée dans une relation amoureuse ; enfin, selon elle, une femme sans conjoint n'est en

12. Yasuda Satsuki, 1914, "Ikiru koto to teisō to : Hankyō kugatsu gō "Taberu koto to teisō to” o yonde » 生きる事と貞操と一反響九月号「食べる事と貞操と」を読んで (Vivre et rester chaste : après lecture de "Manger et rester chaste " du numéro de septembre de Hankyō), Seitō, n 4, vol. 4, décembre 1914 (Orii 1991 : 20-24).

13. Ici, les deux termes apparaissent en tant que synonymes, ce qui montre que les concepts d'aujourd'hui ne s'étaient pas encore imposés à l'époque. Nous pouvons à titre indicatif citer les définitions données dans le Nihongo kokugo daijiten 日本語国語大辞典 (Grand dictionnaire de la langue japonaise) : shojo désignait initialement les femmes célibataires, alors que dōtei désignait une personne n'ayant jamais eu de relation sexuelle. 


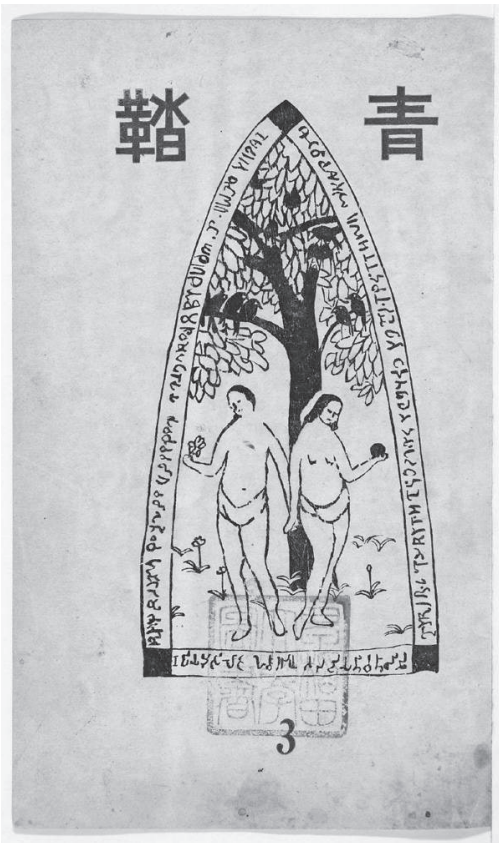

Fig. 1. Couverture de Seitō, numéro de mars 1913, avec un dessin d'Otake Kōkichi (Beniyoshi) 尾竹紅吉. Bibliothèque de l'université Waseda.

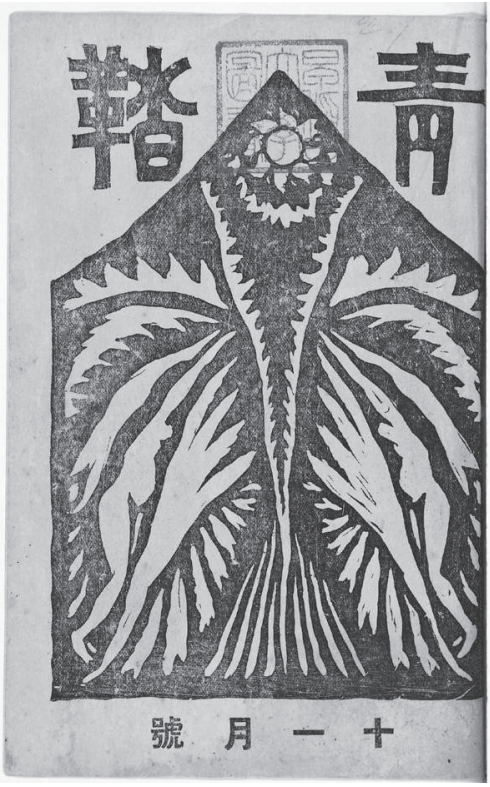

Fig. 2. Couverture de Seitō, numéro de novembre 1914, avec un dessin d'Okumura Hiroshi 奥村博. Bibliothèque de l'université Waseda.

aucun cas liée par une quelconque forme de chasteté, puisque la chasteté naît avec l'amour (Orii 1991 : 40).

Or c'est sur ce dernier point que les deux femmes sont en profond désaccord. Pour Yasuda, la chasteté est une « propriété essentielle à chacun " (Orii 1991 : 44) ${ }^{14}$, inhérente à chaque être humain depuis sa naissance, alors que pour Ikuta, la chasteté naît avec l'amour pour un homme, et se rapproche

14. Yasuda Satsuki, 1915, «Ome ni kakatta Ikuta Hanayo san ni tsuite » お目に懸つた 生田花世さんに就いて (À propos de Mme Ikuta Hanayo que j’ai rencontrée), Seitō, nº 2, vol. 5, février 1915 (Orii 1991 : 43-48). 
ainsi de la fidélité (Orii 1991 : 49) ${ }^{15}$. Par contre, toujours selon Ikuta, la virginité a une importance toute particulière, même si elle ne peut dépasser le poids de la vie, alors que pour Yasuda, la virginité fait partie de la chasteté, laquelle a un poids bien plus important que la vie (Orii 1991 : 52).

Itō Noe participe aux débats en commençant par critiquer la morale relative à la chasteté (teisō) qui établit qu' « une femme ne doit pas avoir deux partenaires dans sa vie ", ou que "seule la chasteté de la femme doit être prise en considération ", etc. ${ }^{16}$. Itō Noe reprend la pensée d'Ikuta Hanayo, pour qui la perte de la virginité est un drame en soi - en ce que la femme ne pourra plus faire un mariage heureux - mais en aucun cas un événement insurmontable. Or, Itō Noe pose ici une question fondamentale : pourquoi la virginité et la chasteté ont-elles une si grande importance aux yeux de la société ? "Pourquoi ne pourrions-nous pas nous marier si nous perdons notre virginité ?" (Orii 1991 : 63). "Tout serait différent si Mme Ikuta ne raisonnait pas en termes de bien et de mal, et ignorait ces valeurs " (Orii 1991 : 62), " il y a tant d'hommes qui perdent leur virginité avant le mariage, la docilité des filles m'exaspère »(Orii 1991 : 63).

Hiratsuka Raichō prit la parole à son tour en mars 1915, en se concentrant notamment sur la signification et sur la valeur de la virginité, shojo ${ }^{17}$. Pour elle, être vierge au mariage est une exigence purement masculine qui trouva un écho également du côté de la femme, chez qui le mariage est la principale garantie de subsistance. Ainsi, la morale, les pratiques, le droit et les institutions seraient-ils le fruit des désirs masculins (Orii 1991 : 67). Le problème pour Hiratsuka Raichō n'est pas la valeur de la virginité ; il faut se demander quand et comment nous devons la perdre (Orii 1991 : 69). Un mariage sans amour est contraire à la chasteté, tout comme un amour spirituel privé de plaisir charnel.

À partir de l'article de Hiratsuka Raichō, les débats se concentrèrent davantage sur la signification de la chasteté. Une série d'articles s'ensuivirent,

15. Ikuta Hanayo, 1915, "Futatabi dōtei no kachi ni tsuite. Yasuda Satsuki sama e " 再び童貞の価值について——安田泉月様へ (De nouveau sur la valeur de la virginité. À l'attention de Mme Yasuda Satsuki), Hankyō, février 1915 (Orii 1991 : 48-57).

16. Itō Noe, 1915, «Teisō ni tsuite no zakkan » 貞操に就いての雑感 (Diverses pensées sur la chasteté), Seitō, n 2, vol. 5, février 1915 (Orii 1991 : 58-65).

17. Hiratsuka Raichō, 1915, “Shojo no shinka » 処女の真価 (La véritable valeur de la virginité), Shinköron, mars 1915 (Orii 1991 : 65-71). 
avec, entre autres, Ōsugi Sakae qui qualifia la chasteté et la sauvegarde de la virginité comme étant une exigence instinctive et sociale ${ }^{18}$; et Harada (Yasuda) Satsuki qui, mariée entre-temps, revient sur la notion de la chasteté. Selon elle, celle-ci appartient à chacun et dépasse largement la simple considération sexuelle, puisque qu'il s'agit d'une exigence à la fois instinctive et rationnelle qui doit s'accomplir - et là, elle reprend les termes de Hiratsuka Raichō - quand le désir charnel et l'amour spirituel se rencontrent, pour les femmes comme pour les hommes ${ }^{19}$. À partir de ce texte, la chasteté masculine devient sérieusement l'un des sujets principaux du débat. Yosano Akiko apporte un point de vue nouveau au débat ${ }^{20}$ : pour elle, la chasteté n'est pas une morale, mais ressemblerait plus à une sorte de richesse dont chaque être dispose en lui-même, et pour lui-même. En renonçant à sa chasteté (virginité), Ikuta se serait juste appauvrie de cette valeur, elle serait " morte du point de vue de la chasteté » (Orii 1991 : 92). Ce n'est en aucun cas le mari qui la possède, "les femmes étant désormais suffisamment fortes et intelligentes " (Orii 1991 : 93). Par contre, si l'homme trompe sa femme, "nous ne sommes plus devant un problème de chasteté, mais un problème d'amour. Alors que la chasteté représente une valeur absolue, l'amour, qui est une sorte de contrat entre deux individus, revêt une valeur relative. Si l'un viole son engagement, l'autre peut très bien abandonner ses propres engagements " (Orii 1991 : 93-94). Or, Yosano Akiko ne distingue pas véritablement la virginité d'une femme non mariée et la chasteté d'une femme mariée, ce qui rend complexe la compréhension de son texte.

Alors que, jusqu’à ce stade, le débat avait principalement été mené par des femmes, voyant la proportion que cela avait pris, des hommes saisirent leur plume à leur tour. Takashima Heizaburō 高島平三郎 (1865-1946) et

18. Ōsugi Sakae, 1915, «Shojo to teisō to shūchi to. Noe-san ni ataete katawara Bakazan o nonoshiru " 処女と貞操と羞恥と一一野枝さんに与へて傍らバ華山を罵る (La virginité, la chasteté et la pudeur. À l'attention de Noe, tout en insultant Bakazan), Shinköron, avril 1915 (Orii 1991: 72-83).

19. Harada Satsuki, 1915, “Teisō no igi to seizon no kachi ni tsuite "貞操の意義と生存 の価值に就いて (Sur la signification de la chasteté et la valeur de la vie), Shinkōron, avril 1915 (Orii $1991: 83-91$ ).

20. Yosano Akiko 1915, “Teisō ni tsuite » 貞操に就いて (Sur la chasteté), Fujo shinbun, $n^{\circ} 783,21$ mai 1915 (Orii 1991 : 92-94). 
Abe Isoo 安部磯雄 (1865-1949) apportèrent un point de vue masculin au débat, le premier en prônant la moralité de la chasteté, qui oblige au respect tant d'un point de vue individuel que social, voire étatique, à la fois pour les femmes et pour les hommes ${ }^{21}$, alors que selon le second - et ce en réponse implicite à Yosano Akiko -, la chasteté est toute relative, naissant du moment où il y a un sentiment d'amour ${ }^{22}$. Le débat continua un moment, animé par différents auteurs hommes et femmes qui s'accordèrent au moins sur un point : la chasteté masculine existe bel et bien, même si, pour certains, elle n'est pas de la même nature que la chasteté féminine.

Le dernier article qui attire notre attention est celui de Hiratsuka Raichō, qui apporte une sorte de conclusion à ce débat ${ }^{23}$. En pointant du doigt la naissance d'une nouvelle morale de la chasteté, Hiratsuka Raichō définit l'ancienne morale sous trois formes, exclusivement féminines : la virginité (shojo) ou la pureté absolue (zettaiteki junketsu 絶対的純潔) pour les femmes avant le mariage ; la chasteté (teisō) pour les femmes mariées; et le veuvage définitif pour les veuves. Selon elle, ces règles sont le fruit d'une longue évolution sociale, et d'un esclavagisme sexuel de la femme. Pour ce qui est de la chasteté des femmes mariées, Hiratsuka avance qu'elle n'est en général pas le résultat d'un amour chez la femme, mais plus d'une peur de sanction sociale qui aurait pour conséquence d'ôter à la femme la possibilité de survie. Chez l'homme, la violation de la chasteté par son épouse serait perçue avant tout comme un vol, puisque la chasteté lui appartient de plein droit. Partant de ce constat, selon Hiratsuka, une nouvelle morale serait sur le point de naître. Désormais, les femmes dites " chastes ", qui vivent sans amour avec leur mari, violeraient au contraire cette nouvelle morale, puisque la véritable chasteté n'existe pas sans véritable amour. Elle conclut par un appel aux femmes à imposer cette nouvelle valeur de la chasteté aux hommes grâce à leur pouvoir décisionnel, en choisissant désormais un

21. Takashima Heizaburō, 1915, "Teisō ni tsuite " 貞操に就いて (Sur la chasteté), Fujo shinbun, $\mathrm{n}^{\circ} 784$ et 786, 28 mai et 11 juin 1915 (Orii 1991 : 95-100).

22. Abe Isoo, 1915, “Watashi no teisōkan" 私の貞操観 (Ma vision de la chasteté), Fujo shinbun, $\mathrm{n}^{\circ} 787,18$ juin 1915 (Orii 1991 : 101-103).

23. Hiratsuka Raichō, 1916, “Sabetsu-teki sei dōtoku ni tsuite " 差別的性道徳に就いて (Sur la morale sexuelle de nature discriminatoire), Fujin kōron, vol. 1, $\mathrm{n}^{\circ} 10$, octobre 1916 (Orii $1991: 122-130$ ). 
homme pour ses valeurs, et non pour sa fortune ou pour son statut social, ce qui ferait évoluer la société.

Le débat sur la chasteté prit ainsi fin, en laissant derrière lui un réel problème de conscience auquel droit et institution durent répondre. L'évolution et la nouvelle définition des mots tels que teiso ot shojo s'imposèrent ainsi au fil du temps, donnant un bel exemple de formation d'un cadre de réflexion commun.

\section{La chasteté, un droit de la personnalité}

À l'image d'Itō Noe qui cria « à bas les coutumes " (shūzoku daha 習俗打 破) (Orii 1991 : 65), les femmes de Seitō s'accordèrent à dire que la société devait évoluer, que les institutions devaient changer, en faveur d'un mariage monogamique issu d'un amour réciproque ${ }^{24}$. Le droit fut alors souvent tenu pour responsable $e^{25}$. Cette position rencontra nombre de critiques, tant de la part des partisans de l'institution de l'ie 家 du Code civil que des militant(e)s de la Société de tempérance des femmes chrétiennes du Japon (Nihon kirisutokyō fujin kyōfūkai 日本キリスト教婦人矯風会) pour qui la monogamie devait être respectée dans l'intérêt de l'État (Iwabuchi 1998 : 313). Or, l'important fut avant tout de saisir le sens de la chasteté. En effet, les mots teisō et shojo avaient une grande diversité de sens. Par exemple, dans certaines régions, shojo désignait tout simplement les filles célibataires - qu'elles soient vierges ou non -, tandis que teisō pouvait se limiter au temps d'un mariage, la chasteté n'étant exigée ni avant, ni après (Muta 2009 : 224).

24. Voir les articles de Christine Lévy, de Marion Saucion et d'Ōta Tomomi dans ce dossier. Sur la notion de ren.ai 恋愛, voir Butel (2011).

25. Cela dit, nous pouvons constater que l'institution de l'ie 家 (maison) était devenue un symbole de l'inégalité, à tel point qu'elle pût être mal interprétée. À titre d'exemple, Ikuta Hanayo critique l'interdiction aux femmes de posséder (shoyū 所有) tout bien (Ikuta 1914, cf. Orii 1991 : 17). Cette assertion n’est pas tout à fait juste, dans la mesure où les femmes, comme les hommes, possédaient leur patrimoine qu'elles soient mariées ou non, l'incapacité de la femme mariée ne touchant que la gestion des biens. Voir Konuma (2011). 
Or, comment ce concept, qui relève principalement de la morale, s'infiltra-t-il dans le domaine juridique ? Comment le droit incorpora-t-il en son sein des valeurs à la base relevant de la morale ?

Pour répondre à cette question, il est nécessaire d'analyser le droit civil, notamment les dispositions relatives au mariage et au divorce, afin de décrire le contexte dans lequel le débat est né, ainsi que les évolutions législatives et judiciaires qui s'ensuivirent. Le point de départ est incontestablement le Code civil. Entrées en application en 1898, et jusqu'à leur suppression en 1946, les dispositions relatives à la famille figuraient dans le quatrième livre (sur la parenté, shinzoku 親族) et le cinquième livre (sur la succession, sōzoku 相続), les deux livres constituant le droit des statuts (mibunhō 身分 法 $)^{26}$. Trois points seront successivement abordés : la définition de la chasteté, le poids de la virginité et la chasteté masculine.

\section{a. La définition de la chasteté}

Ume Kenjirō 梅謙次郎 (1860-1910), l'un des rédacteurs du Code, commenta dans un ouvrage, article par article, les dispositions du Code. Cet ouvrage constitue une base précieuse pour contextualiser et interpréter les dispositions. Ici, le mot teisō apparaît à deux reprises, non dans les dispositions du Code, mais dans les commentaires d'Ume Kenjirō sur les effets du mariage (Ume 1901 : 142-144) :

"Les effets du mariage relèvent essentiellement du domaine de la morale : il s'agit par exemple du respect de la chasteté (teisō) tant par l'homme que par la femme ; du respect de l'obligation d'entraide ; ou de l'obligation pour l'homme de protéger la femme, et l'obligation pour la femme de respecter l'homme. Certes, selon les pays, les devoirs moraux se trouvent incorporés dans le droit. Pour ma part, je suis convaincu que cela n'est pas approprié, même si la violation de certains devoirs doit juridiquement être sanctionnée : en cas de violation de la chasteté (teisō) par l'épouse, non seulement le droit pénal la condamnerait, mais sur le plan civil aussi, puisque l'époux pourrait demander le divorce. Ainsi, si l'épouse causait du tort à l'époux, elle devrait le dédommager. Les époux ont l'obligation de vie commune

26. Depuis la grande réforme d'après-guerre qui supprima l'ensemble des règles relatives au statut individuel au sein de l'ie, ces deux livres sont connus sous la dénomination commune du droit de la famille (kazokuhō 家族法). 
(article 789), une obligation qui résulte du devoir d'entraide ; ils ont également l'obligation réciproque de subvenir aux besoins de l'autre conjoint (article 790) ; ou encore, l'époux, en ce qu'il doit à l'épouse sa protection, devient tuteur de son épouse mineure (article 791), et l'épouse, en ce qu'elle doit respect à l'époux, est dans l'obligation de recueillir son accord pour accomplir tout acte juridique (article 14). Ces devoirs sont certes formulés dans le présent code, mais je considère qu'il n'est pas nécessaire d'intégrer d'autres devoirs moraux dans le domaine juridique. »

Comme ce passage le montre clairement, le mot teisō est utilisé pour désigner la chasteté, au sens de fidélité, au sein du mariage. La chasteté est donc un des engagements matrimoniaux, elle est sanctionnée par un divorce en cas de violation, du moins en cas d'adultère féminin. Notons que ce divorce pour adultère ( $k a n t s \bar{u}$ 葌通) figurait parmi les dispositions relatives au divorce judiciaire, la règle de base étant le divorce par consentement mutuel, une procédure moins lourde, nécessitant une simple déclaration de divorce à la mairie. En cas de désaccord, les juges étaient donc saisis pour dire si le divorce pouvait être prononcé. Pour cela, ils se référaient aux motifs du divorce énumérés exhaustivement dans l'article $813^{27}$. Cela laissait finalement très peu de marge aux juges, même si le mariage présentait des signes de faillite.

Dans le cadre du divorce judiciaire, le Code civil sanctionnait l'adultère féminin plus sévèrement que l'adultère masculin (article 813) : un époux trompé pouvait demander le divorce simplement du fait de la violation de la chasteté par son épouse, alors qu'une épouse trompée ne pouvait demander le divorce que si son époux avait été condamné pénalement pour "relations malsaines"(kan.inzai 䒛淫罪). Ces relations dites malsaines, au nombre de trois, comptaient le viol, la relation avec mineure de moins de treize ans et la relation adultérine avec une femme mariée (Code pénal,

27. Outre la violation de la chasteté de l'épouse, ou la condamnation pour « relation malsaine " de l'époux, le Code prévoyait comme motifs de divorce la polygamie, la condamnation du conjoint à une peine supérieure à trois ans d'emprisonnement, la maltraitance des beaux-parents, la maltraitance par les beaux-parents, l'abandon du conjoint, la disparition de plus de trois ans et l'annulation ou la dissolution des liens d'adoption lorsque le mari était à la fois marié avec la fille héritière et adopté par ses beaux-parents (muko yōshi 婿養子). Ces motifs se référaient donc à une existence de faute d'un des conjoints (divorce pour faute ou divorce-sanction), un simple constat de faillite (divorce-faillite) ne suffisant pas en cas de désaccord des époux. 
article 183). L'exigence d'une condamnation pénale et d'une circonstance aggravante pour le cas de l'adultère masculin limitait de fait considérablement l'obligation de chasteté chez l'homme.

Ainsi, la chasteté semble bien délimitée dans le Code civil : il s'agit d'une exigence purement conjugale et inégalitaire entre époux. Cependant, exceptionnellement, la chasteté pouvait s'imposer au-delà du mariage. C'est le cas de l'interdiction de se remarier avec le partenaire adultérin en cas de divorce judiciaire pour adultère (article 768). L'amour, tant encouragé par les femmes de Seitō, et placé au centre de la nouvelle morale de la chasteté, étant en l'occurrence sanctionné au nom de la chasteté, comprise dans le sens de la fidélité.

Malgré cette interprétation restreinte du devoir de chasteté sur le plan législatif, le mot teisō continuera à revêtir différentes significations. Dans l'un des rares ouvrages juridiques d'avant-guerre portant sur la chasteté, Ōsawa Ichiroku 大澤一六 (1886- ?), avocat, commence son analyse en pointant du doigt la complexité du concept de chasteté. L'auteur effectua pour cela un sondage auprès des étudiantes de l'université, en leur demandant ce que signifiait pour elles le mot teisō. Il recueillit alors plusieurs réponses : il s'agit de "protéger la virginité ", de " rester fidèle à son époux, sans avoir de relation sexuelle avec un autre homme », ou encore de "rester chaste après le décès de son époux, sans céder à d'éventuelles tentations ", etc. (Ōsawa 1934 : 10). Ces interprétations montrent que la notion de la chasteté peut porter sur une période indéfinie pour certains, dépassant largement le cadre du mariage, alors que pour d'autres, il s'agirait d'une règle régissant le mariage. La chasteté a donc à la fois une portée sur le plan juridique, mais aussi sur le plan de la morale, les deux n'étant pas toujours en parfait accord, comme le révèle de façon éloquente ce sondage.

L'auteur tente néanmoins de proposer une définition de la chasteté :

"La chasteté (teisō) est un droit de la personnalité (jinkaku-ken 人格権) que détient toute femme dans le domaine de la sexualité, afin de se protéger fermement et de rester propriétaire d'un corps et d'une âme inviolés. Par conséquent, elle est sujette à une obligation de ne pas s'engager simultanément auprès de plusieurs hommes, du moment où elle se donne à un homme. Ce droit et cette obligation constituent, ensemble, la base des relations conjugales, et par conséquent, des dispositions juridiques. " (Ōsawa 1934 : 17). 
Il faut noter que la chasteté est ici qualifiée de droit de la personnalité, soit un droit inhérent à tout être humain, inaliénable, relatif à l'intégrité physique $^{28}$. Or, en analysant le contenu de ce droit, nous nous rendons rapidement compte que ce n'est qu'en apparence qu'il est question de protection de la femme. En effet, il met en avant un droit de la femme de rester chaste (qui est une forme de liberté décisionnelle) sans pour autant couvrir l'aspect négatif de ce droit, celui de ne pas rester chaste par exemple, un droit qui va de la naissance jusqu'à la mort, alors que l'obligation, elle, se limite à un temps précis, celui d'un engagement conjugal.

\section{b. Le poids de la virginité}

La virginité ou l'absence de celle-ci ne faisait en aucun cas l'objet d'une disposition, tant pour la conclusion que pour l'annulation ou la dissolution du mariage. Pour autant, pourrait-on en conclure qu'elle fut dépourvue de toute valeur juridique ? En revisitant les décisions judiciaires de Taishō et du début de Shōwa, nous nous rendons rapidement compte que la virginité continuait à avoir une certaine portée sociale, ce qui donne une dimension bien réelle aux débats de Seitō. L'ouvrage de Yamaguchi Yohachirō, avocat, est extrêmement révélateur : il propose un recueil de décisions judiciaires relatives à la chasteté des femmes (Yamaguchi 1936). Comment les juges rendaient-ils leur décision en marge de dispositions législatives ? Nous pouvons percevoir la position protectrice des juges, notamment à travers deux affaires où il fut question de monnayer la valeur de la chasteté.

La première affaire concerne une promesse de mariage annulée par l'homme alors que les deux ex-fiancés avaient eu des rapports sexuels (Yamaguchi 1936 : 311-319). La femme, s'estimant avoir été violée dans sa chasteté, porte plainte, alors que l'homme avance la non violation de la chasteté puisque la femme n'était pas vierge au moment des faits. La question se posa en ces termes : une promesse de mariage non tenue par

28. À la même période, Yamaguchi Yohachirō 山口與八郎 propose pour sa part une définition du droit de la personnalité : il s'agit d'un droit "inhérent à la vie humaine ", l'homme, " tant qu'il est en vie, ayant un honneur et une confiance à sauvegarder, et de ce fait, détenant une dignité. [...] Même si l'on n'est plus vierge, chaque être dispose d'une dignité en ce qui concerne sa chasteté. » (Yamaguchi 1936 : 309). 
l'homme, alors que le mariage fut en soi consommé, est-elle de nature à sanctionner celui-ci pour violation de la chasteté, même si l'ex-fiancée n'était plus vierge au moment des faits ? Autrement dit, la femme peut-elle prétendre à la violation de son droit de chasteté alors qu' elle avait déjà perdu sa virginité ? Laffaire fut traitée par le tribunal de district de Yokohama qui rendit son jugement le 17 août 1930. Les juges, en formulant ainsi leur motif, donnèrent gain de cause à la femme : " même si la femme n'était plus vierge, cela ne lui ôte pas pour autant le droit d'être dédommagée, bien que cette donnée puisse influencer le montant du dédommagement ». Il en ressort que la chasteté a été définie par les juges, du moins dans le cadre de cette affaire, comme étant un droit de la femme à rester fidèle à un homme dans le cadre d'un mariage. En précisant ainsi la notion de la chasteté, les juges restèrent dans la conception du Code civil et écartèrent la définition avancée par l'ex-fiancé, pour qui la chasteté était l'équivalent de virginité. Or, le point particulièrement intéressant est que la valeur de la chasteté dépendait de la virginité et de l'âge de la femme. En tenant compte de ces deux éléments - la femme avait vingt-quatre ans au moment des faits -, les juges fixèrent le montant des dommages-intérêts à 500 yens $^{29}$.

Cette position est également partagée dans une autre affaire rendue par la cour d'appel d'Osaka (date inconnue). En l'espèce, une serveuse de café avait partagé la vie d'un fils issu d'une famille riche, avec qui elle eut une promesse de mariage. Or, ce dernier mit fin à cette relation en septembre 1927. À la demande de dédommagement, l'ex-fiancé avança l'absence de chasteté de la femme du fait de la nature de son travail et de nombreuses relations qu'elle avait eues avec d'autres hommes. Or, en l'absence de preuve, les juges donnèrent gain de cause à l'ex-fiancée et condamnèrent l'ex-fiancé à 700 yens de dommages-intérêts (Yamaguchi 1936 : 349-358).

En citant ces affaires, Yamaguchi conclut que les femmes non vierges, ou encore les femmes réputées pour leurs mœurs légères, ont également le droit d'invoquer leur chasteté, bien que la valeur de celle-ci dépende de différents critères tels que la virginité. Malgré ce constat, les explications de Yamaguchi peuvent paraître déplacées par endroit, reflétant bien l'état

29. Selon l'indice des prix à la consommation, le montant équivaut à environ 900000 yens aujourd'hui, soit 9000 euros. 
d'esprit de certains juristes pour qui la virginité restait un élément central, et la chasteté de la femme la propriété de l'homme :

«Pour déterminer la valeur [de la chasteté], il est fréquent que la virginité et la nonvirginité créent une différence, puisque la valeur d'un objet n'est en aucun cas égale selon son âge. En effet, à moins qu'il s'agisse d'une antiquité, un vieil objet est, en principe, d'une moindre valeur qu'un nouvel objet. La règle est particulièrement vraie pour ce qui est de la chasteté. Toutefois, dans ce cas, la chasteté garde une valeur, même si elle en est réduite." (Yamaguchi 1936 : 310).

\section{c. La chasteté masculine}

Enfin, un dernier point du débat attire notre attention. Il s'agit de l'égalité des sexes dans la chasteté et de la protection de la femme au sein du mariage. En plein milieu du débat, précisément le 26 janvier 1915, un arrêt historique fut rendu par la Haute Cour de justice. Cet arrêt est qualifié de révolutionnaire du point de vue jurisprudentiel et du droit de la famille, mais aussi fondateur du point de vue de la sociologie juridique, puisqu'il prit clairement position en faveur de la protection d'une épouse de fait c'est-à-dire mariée socialement à un homme sans que le mariage ne soit déclaré juridiquement à la mairie -, abandonnée sans raison valable par son mari. Ainsi, au mariage de fait (naien 内縁) fut appliquée la théorie du "semi-mariage" (junkon 準婚) afin d'assurer certaines protections en cas de rupture abusive des liens (Seizelet 2001). Même si la question ne concerne pas directement la chasteté, cet arrêt reflète bien la tendance de l'époque, soit la protection de la partie faible par les juges et la prise en considération de la réalité sociale pour ne pas rester enfermé dans une interprétation exégétique des dispositions législatives.

Par ailleurs, dans le monde des juristes, un débat sur l'adultère avait éclaté dix ans après l'élaboration du Code civil, mettant notamment à contribution les magistrats. Le débat fut lancé en 1908, suite à un article d'Ogawa Shigejirō 小河滋次郎 (1864-1925), spécialiste de la politique sociale, qui remettait en cause la différence de traitement des adultères féminin et masculin. Ogawa critiquait l'article 183 du Code pénal comme étant une injustice pour les femmes, et soutenait qu'il fallait soit sanctionner de la même façon l'adultère masculin, soit supprimer complètement la sanction pour adultère. À ces propos, Katō Hiroyuki 加藤弘之 (1836-1916) réagit pour proposer d'introduire la même sanction aux hommes car, selon 
lui, l'adultère féminin devant être sanctionné à tout prix pour garantir la transmission du sang, sa suppression n'était pas envisageable (Katō 1915). Le débat se poursuivit dans le Hōritsu shinbun 法律新聞 (Journal du droit), notamment par les magistrats autour de l'interprétation de l'article 813 (Murakami 2003 : 164-177). Même si ce débat n'eut pas de suite sur le plan législatif ${ }^{30}$, il eut le mérite de lancer une réflexion chez les juristes sur l'égalité sexuelle dans la chasteté, et ce peu de temps avant notre débat autour de Seitō.

Il a fallu attendre un arrêt de 1927 pour voir la chasteté masculine prendre une réelle place parmi les engagements matrimoniaux. Cet arrêt doit être placé dans son contexte : un an plus tôt, la Commission provisoire de réforme législative ${ }^{31}$ s'était réunie autour de la question de la chasteté masculine, ce qui aboutit à un projet de modification du Code civil, qui introduisit une plus grande obligation de chasteté chez l'homme dans le cadre du mariage. Cette commission proposait de modifier l'article 813 relatif au divorce judiciaire : le divorce serait désormais possible sans passer par une condamnation pénale, en présence d'une " mauvaise conduite extrême (ichijirushiku fugyōseki naru toki 著シク不行跡ナルトキ) du mari ${ }^{32}$ ». Sans introduire une parfaite égalité, cette proposition tentait d'atténuer l'inégalité sexuelle dans l'exigence de la chasteté au sein du mariage.

La tentative de réforme ayant échoué, ce sont les juges qui se mobilisèrent pour reconnaitre la chasteté masculine. L'affaire concernait un homme qui avait abandonné son épouse au profit d'une autre femme, alors que trois enfants étaient nés du mariage. La mère de l'épouse confia à un tiers la mission d'obtenir de l'homme et de sa maîtresse des dommagesintérêts ainsi que des frais d'éducation des enfants, en les menaçant de

30. Critiquer les dispositions législatives relatives au mariage ou au divorce était assimilé à des critiques institutionnelles, et pouvait provoquer une condamnation pour blâme de la part des autorités publiques (Toshitani 1982 : 294-295).

31. Cette commission fut initialement formée pour renforcer l'institution familiale. Pourtant, elle finit par céder devant l'élan démocratique de Taishō, en faveur d'une autorité patriarcale moins rigide et de l'égalité des membres au sein de la famille.

32. Cette formule n'indique pas clairement si l'adultère en fait partie. En laissant une marge de manœuvre, tout en conservant une inégalité sexuelle, elle permettait un compromis. 
porter plainte pour adultère. Or, c'est le mari et sa maîtresse qui portèrent plainte contre ce tiers pour chantage, lequel fut condamné en première instance et en appel. Saisie de cette affaire, la Haute Cour de justice rendit un arrêt le 17 mai 1927, dans lequel elle innocenta ce tiers en reconnaissant que l'épouse avait effectivement droit à des frais d'éducation et rejeta la qualification de chantage. Et pour cela, la Cour précisa :

"L'épouse a certes l'obligation de rester chaste, mais l'époux est également tenu par cette obligation. Même si l'article 813 al. 3 du Code civil ne fait pas de l'adultère de l'époux l'un des motifs de divorce, même si l'article 183 du Code pénal ne sanctionne pas l'adultère masculin, ces dispositions, résultant principalement d'une politique législative particulière issue d'anciennes coutumes, ne peuvent pas empêcher l'épouse d'exiger sur le plan du droit civil la chasteté à son mari. " (Toshitani $1982: 302)$.

\section{Conclusion}

Ainsi, la chasteté, dessinée initialement comme une obligation conjugale à caractère inégalitaire, devint progressivement une obligation également masculine. Cette définition se rapproche de celle d'Ikuta Hanayo, pour qui la chasteté accompagne la vie du couple, à la différence près que le point de départ n'est pas le mariage mais l'amour. Simultanément, la chasteté est devenue un droit de la personnalitée ${ }^{33}$, qui ne dépend pas de la virginité ni du mariage, puisqu'il est reconnu à chaque être le temps de sa vie. Nous pouvons retrouver cette dernière définition dans les propos de Yasuda Satsuki, pour qui la chasteté est inhérente à chaque être, dès sa naissance, jusqu’à sa mort. Il est difficile de dire l'impact réel du débat relatif à la chasteté sur ces différentes évolutions juridiques. Or, le fait est que les femmes de Seitō avaient pressenti l'évolution du concept de la chasteté, conçu initialement comme un devoir inégalitaire, vers un devoir commun aux époux, puis vers un droit de la personnalité. Notons par ailleurs que l'ère Taishō avait ouvert de nouvelles perspectives en termes d'études juridiques, avec l'introduction

33. Voir Kuriu (1929), en particulier son sixième chapitre, "Teisō no shihōteki hogo » 貞操の私法的保護 (La protection en droit privé de la chasteté). 
de la libre science du droit, du droit marxiste et de la sociologie du droit ${ }^{34}$. Ainsi l'époque était-elle propice à de nouvelles interprétations du droit, la méthode exégétique étant à bout de souffle $\mathrm{e}^{35}$.

En ce qui concerne la chasteté, il semble important de distinguer deux aspects : une exigence morale, et une exigence juridique. Le système juridique présentait certes des inégalités sexuelles. Pourtant, les rédacteurs du Code civil, dont notamment Ume Kenjirō, regrettaient cette inégalité avant même son application. Pour Ume, il était évident que la chasteté était un devoir réciproque entre époux, un devoir moral, qui n'avait pas lieu d'être formulé en tant que règle de droit. Le droit devait, selon lui, se concentrer sur les conséquences de ce devoir, soit l'adultère, qui devait être sanctionné sévèrement tant pour la femme que pour l'homme. Or, si le droit a entretenu une inégalité sexuelle, c'est pour des raisons d'ordre coutumier qui laissent " de graves injustices en écartant l'époux de ce devoir alors que la pratique des concubines (mekake 妾) n'a plus lieu d'être dans une société évoluée comme en Occident ». Il poursuit : « je suis convaincu qu'une telle injustice est vouée à disparaître dans un avenir proche »(Ume 1901 : 216).

Il a fallu attendre la grande réforme du Code civil de 1946 pour voir les vœux d'Ume exhaussés. Il n'en demeure pas moins qu'en dissociant droit et morale, les juristes n'ont pas véritablement simplifié les choses. En effet, cela leur permit de maintenir une inégalité juridique, en reléguant à la morale le soin d'introduire une égalité sexuelle. Or, c'est précisément la subsistance de cette " ancienne morale " que critiquèrent les femmes de Seitō, conscientes que son évolution ne dépendait que de l'éveil des femmes.

34. Suehiro Izutarō 未広㛜太郎 (1888-1951) est considéré comme le père de la sociologie du droit au Japon. Dès les années vingt, il se référa à la théorie d'Eugen Ehrlich, la " loi de vie", selon laquelle les normes juridiques ne sont pas toujours en adéquation avec les règles de la vie sociale.

35. Cette méthode, préconisée au XIX ${ }^{\mathrm{e}}$ siècle en France, consistait à interpréter le droit en se référant à l'esprit du texte, représenté dans la volonté des législateurs. 


\section{Bibliographie}

\section{BUTEL Jean-Michel 2011}

« Des couples aimants pour une nation moderne : un nouveau modèle familial dans le Japon de la fin du xix siècle », in GALAN Christian et LOZERAND Emmanuel (dir.), La Famille japonaise moderne (1868-1926). Discours et débats, Arles, Philippes Picquier, pp. 361-378.

\section{DODANE Claire 2000}

Yosano Akiko, poète de la passion et figure de proue du féminisme japonais, Paris, POF.

\section{GALAN Christian}

\& LOZERAND Emmanuel (dir.) 2011 La Famille japonaise moderne (1868-1926). Discours et débats, Arles, Philippes Picquier.

\section{IWABUCHI Hiroko 岩淵宏子 1998} «Sekushuaritī no seijigaku e no chōsen » セクシュアリティーの政治学への挑戦 (Défi dans les études politiques de la sexualité), in Shin-feminizumu hihyō no $\mathrm{kai}$ 新・フェミニズム批評の会 (Association pour une nouvelle critique féministe) (éd.), Seitō o yomu『青鞮』を読む (Lire Seitō), Tokyo, Gakugei shorin 學藝書林, pp. 305-331.

\section{KATŌ Hiroyuki 加藤弘之 1915} «Kantsū ni tsuite » 姦通二付テ (À propos de l'adultère), Hōgaku kyōkai zasshi 法 学協会雑誌 (Revue de l'association du Droit), 26 (1) : 1-6.

\section{KONUMA Isabelle 2011}

"Le statut juridique de la femme mariée pendant l'ère Meiji : inégalité, protection et reconnaissance ", in GALAN Ch. et LOZERAND E., op. cit., pp. 391-409.

\section{KURIU Takeo 栗生武夫 1929} Jinkakuken-hō no hattatsu 人格権 法の発達 (Évolution du droit de la personnalité), Tokyo, Kōbundō shobō 弘文堂書房.

MURAKAMI Kazuhiro 村上一博 2003 Nihon kindai kon.in hōshiron 日本近代婚 姻法史論 (Histoire du droit matrimonial moderne au Japon), Kyoto, Hōritsu bunkasha 法律文化社.

MUTA Kazue 牟田和恵 2009 "Senryaku to shite no onna : Meiji, Taishō no "onna no gensetsu" o megutte» 戦略としての女一一明治・大正 の「女の言説」を巡って (La femme en tant que stratégie : autour des « paroles des femmes » de Meiji et Taishō), in AMANO Masako 天野正子 et alii (dir.), Shinpen Nihon no feminizumu 新編 日本のフェミニ ズム (Le féminisme au Japon [Nouvelle édition]), vol. 3, Tokyo, Iwanami shoten 岩波書店, pp. 211-230.

\section{ORII Miyako 折井美耶子 1991}

Sei to ai o meguru ronsō

性と愛をめぐる論争 (Débats sur le sexe et l'amour), Tokyo, Domesu shuppan ドメス出版. 
ŌSAWA Ichiroku 大澤一六 1934

Teisō no hōritsu 貞操の法律 (Le droit

de la chasteté), Tokyo, Daikyōsha 大京社.

\section{SEIZELET Eric 2001}

"Contra legem ou para legem?

La coutume de l'union matrimoniale de fait en droit japonais avant 1945 », Extrême-Orient Extrême-Occident, 23 : 109-124.

\section{SUZUKI Michiko 2010}

Becoming Modern Women. Love and Female Identity in Prewar Japanese Literature and Culture, Stanford, Stanford University Press.

TOSHITANI Nobuyoshi 利谷信義 1982 《Danshi teisō gimu ronsō » 男子貞操 義務論争 (Le débat sur l'obligation de chasteté masculine), in KATŌ Ichirō 加藤一郎 (dir.), Minpō-gaku no rekishi to kadai 民法学の歴史と課題 (L'histoire et les problèmes du droit civil), Tokyo, Tokyo daigaku shuppankai 東京大学出版会.

UME Kenjirō 梅謙次郎 1901

Minpō yōgi 民法要義 (Commentaires du Code civil), vol. 4, Tokyo, Wafutsu hōritsu gakkō 和仏法律学校.

\section{YAMAGUCHI Yohachirō 山口與八郎} 1936

Teisō mondai to saiban 貞操問題と裁判 (La chasteté et les procès), Tokyo, Meiji daigaku shuppanbu 明治大学出版部. 